\title{
Influence of the Current Density and Resistivity on the Optical Properties of P-Type Porous Silicon Thin Films Fabricated by the Electrochemical Anodizing Method
}

\author{
J. Torres, F. Castillejo, and J. E. Alfonso \\ Grupo de Física de la Materia Condensada, Departamento de Física, \\ Universidad Nacional de Colombia, Bogotá, Colombia
}

Received on 8 December, 2005

\begin{abstract}
Porous Silicon thin films were produced in this work by the electrochemical anodizing method. The samples were fabricated anodizing $\mathrm{p}$ type Si substrates with different resistivities in hydrofluoric acid. Samples were prepared at different current densities. The films were characterized through reflectance and room photoluminescence (PL) measurements in the visible region. A simple theoretical model is proposed to calculate the Optical constants of the material using a theoretical simulation of the reflectance spectra. The maximum positions of PL signals are shifted to long wavelengths as the porosity increase in samples prepared with substrates of resistivities of 0.1 and $1 \Omega-\mathrm{cm}$, while an opposite behaviour is observed in samples prepared on more doped substrates. The optical constants obtained through the calculation are the spectral variation of the refractive index and extinction coefficient, also are obtained the sample thickness and surface roughness.
\end{abstract}

Keywords: Porous silicon; Photoluminescence, Optical properties; Reflectance; Optical absorption

\section{INTRODUCTION}

Since the discovery of photoluminescence in porous silicon, this material has been intensively studied with the purpose of explaining its visible light emission capability, to optically integrate it into the widely established Silicon technology.

It is of great importance to obtain the optical constants of the material because parameters such as the absorption coefficient and refraction index cooperate in the interpretation and control of its emission mechanisms. However, optical characterization of the material presents a certain degree of difficulty not only in the experimental part but in the interpretation of the results as well. The material is supported on a thick and visibly opaque substrate which restricts the measurement techniques for reflectance and ellipsometry [1-3], or the preparation of auto standing thick samples; additionally, the surface is rugged, leading to the presence of an important component of diffused light in the spectra that must be taken into account in the calculation process.

This work presents results on photoluminescence of samples prepared at different conditions and a calculation method that allows determining, in a simple way, the optical properties of porous silicon thin films, from the theoretical simulation of reflectance spectra.

\section{EXPERIMENTAL DETAILS}

The porous silicon (PS), studied in this work, was prepared by the Galvanostatic anodizing method of silicon wafers.

Samples were prepared using (100), p-type (Boron) silicon substrates with resistivities of $0.01,0.1$ and $1 \Omega-\mathrm{cm}$, The electrolyte used was made up by Hydrofluoric acid (HF) at a $20 \%$ $(\mathrm{p} / \mathrm{v})$ concentration in isopropyl alcohol (IPA). Anodizing currents densities of $1.3,3.8,6.4,8.9$ and $11.5 \mathrm{~mA} / \mathrm{cm}^{2}$ were used during $60 \mathrm{~min}$. After the anodizing process, the samples were rinsed three times with de-ionized distilled water and dried out with gaseous nitrogen for 1 to 3 minutes.

Porosity was measure using a gravimetric method with an error of $10 \%$

Room temperature photoluminescence (PL) was produce using a $332 \mathrm{~nm}$ line from a high power Hg lamp. Next the PL signal was passed through a long pass filter (cut off 500 $\mathrm{nm}$ ), analized by an ORIEL Corner Stone 1/4 m monocromator, detected by an ORIEL photomultiplicator tube (77348) and processed by a LocK-In amplifier. Each sample was prepared two times and photoluminescence measurements were performed several times in order to guarantee the photoluminescence behavior.

Reflectance measurements in the visible region (350-1000 nm) were taken using a Perkin Elmer, Lambda 2 spectrophotometer with integrating sphere. The light beam was almost perpendicular to the sample surface.

Substrate and equipment response are subtracted from the sample spectra.

\section{RESULTS AND DISCUSSION}

\section{A. Photoluminescence}

Figure 1 presents the porosity obtained using weight measurements together with the thickness obtained trough calculation. It is observed that porosity increase as the current density does but an opposite behavior is observed when the doping concentration is increased.

Figure 2 presents normalized photoluminescence curves obtained in samples of $0.01 \Omega$-cm resistivity, prepared at different anodizing currents (anodizing time: 60 minutes). The spectra are wide and a dependence on the anodizing current for the spectral position of the maximum photoluminescence signal has been found. In these samples the position of the maximum shifts toward shorter wavelengths as the anodizing current (porosity) increases.

This result agrees with some other authors predictions [4] 


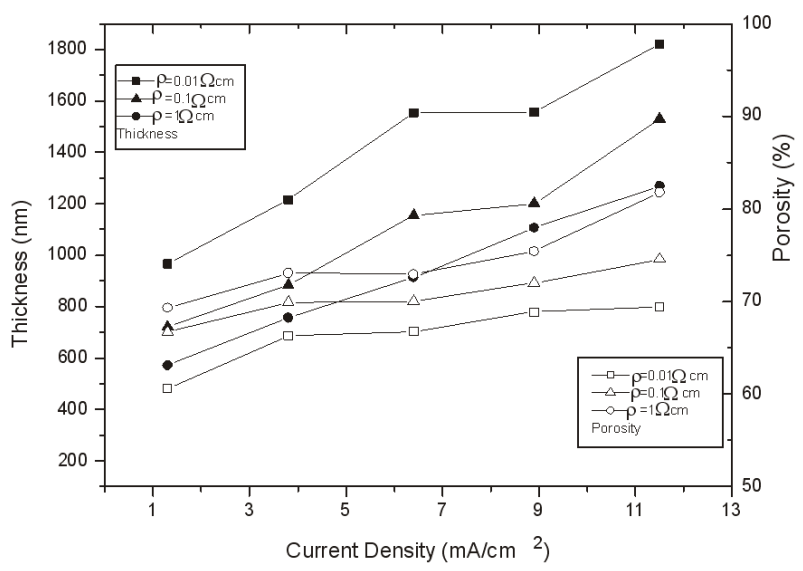

FIG. 1: Porosity and thickness of samples. Porosity was obtained trough a gravimetric technique and thickness was obtained trough calculation.

stating there must be a shift toward the blue as the quantum dimension of the skeleton in the sample, diminishes. A different behavior is observed in the samples prepared on substrates of resistivities 0.1 and $1 \Omega$-cm vs. $0.01 \Omega$-cm, figures 3 and 4 ; the photoluminescence maximum shifts toward longer wavelengths as the anodizing current increases (porosity increases), so the hypothesis of the shift toward the blue as the sample porosity increases, is contradicted in these samples. This result indicates that the porous silicon emission is strongly dependent on the sample fabrication conditions and the substrate resistivity.

\section{B. Calculation model used for the reflectance measurements}

The samples prepared at 60 minutes anodizing time did not present interference fringes in the reflectance spectra because of their great, about $20 \mu \mathrm{m}$, thickness. The calculation model for the optical properties of the material proposed in this work requires the presence of maxima and minima in the spectra; samples with 6 minute anodizing time were prepared to remedy this.

Figure 5 Shows an experimental reflectance spectrum as a function of the incident radiation wavelength, obtained for a porous silicon sample prepared at a 6 minute anodizing time, $\mathrm{HF}$ at a $20 \%(\mathrm{p} / \mathrm{v})$ concentration in (IPA), substrate resistivity $1 \Omega-\mathrm{cm}$ and $11.5 \mathrm{~mA} / \mathrm{cm}^{2}$ current density.

The spectrum clearly presents two regions: one showing the interference fringes between 500 and $1000 \mathrm{~nm}$, named in this work low absorption region, and the high absorption one between 350 and $500 \mathrm{~nm}$, where the intensity of the fringes notably decreases or disappears altogether.

The existence of interference fringes permit to conclude that the sample, despite its disorder, has two defined interfaces air/PS and PS/Si, that the latter are flat and parallel in such way that it is possible to consider the porous silicon thin film as coming from a material with plane and parallel interfaces with a refraction index that is not of air or silicon but of

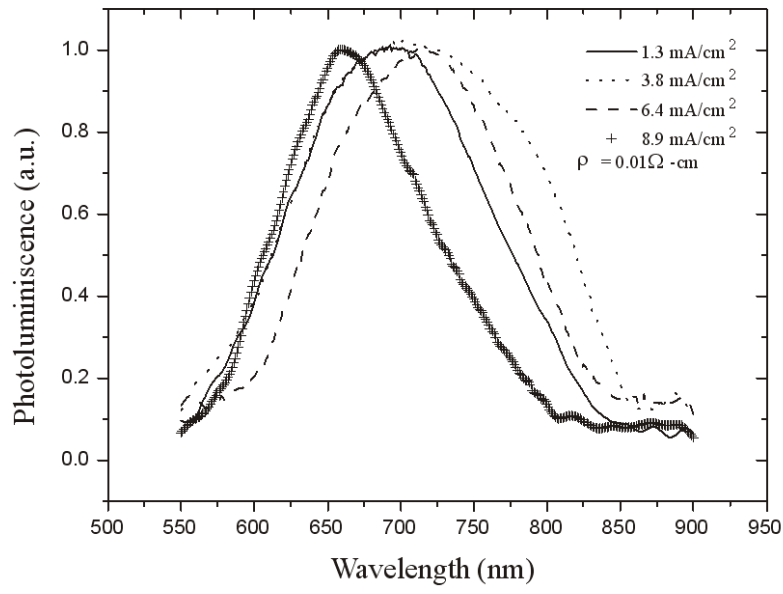

FIG. 2: Photoluminescence spectra of samples prepared on substrates of resistivity $0.01 \Omega$-cm.

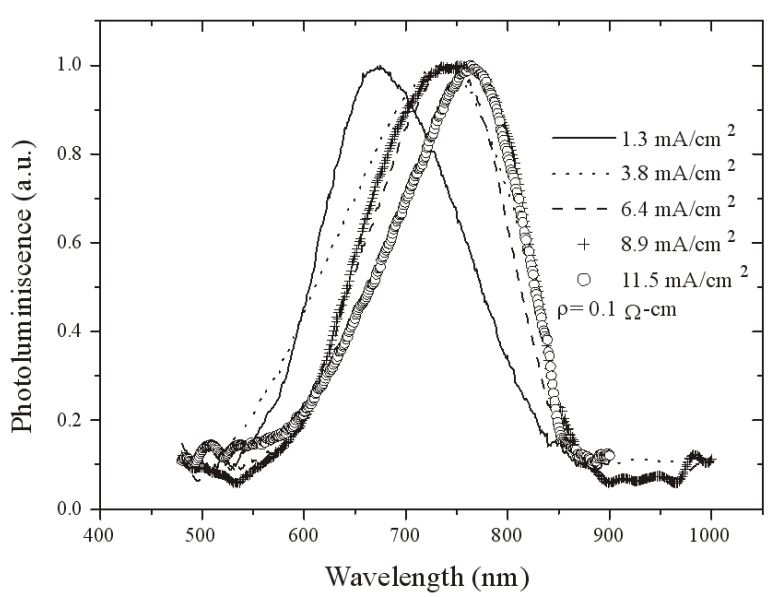

FIG. 3: Photoluminescence spectra of samples prepared on substrates of resistivity $0.1 \Omega$-cm.

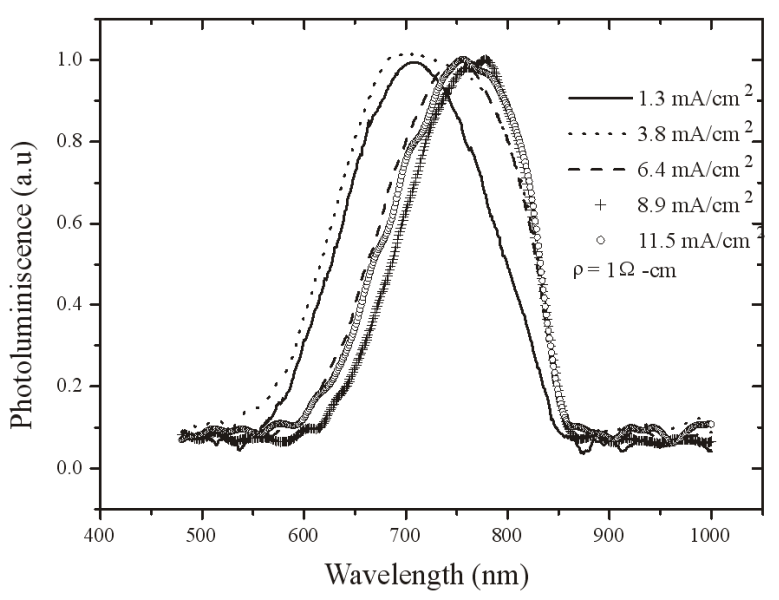

FIG. 4: Photoluminescence spectra of samples prepared on substrates of resistivity $1 \Omega$-cm. 


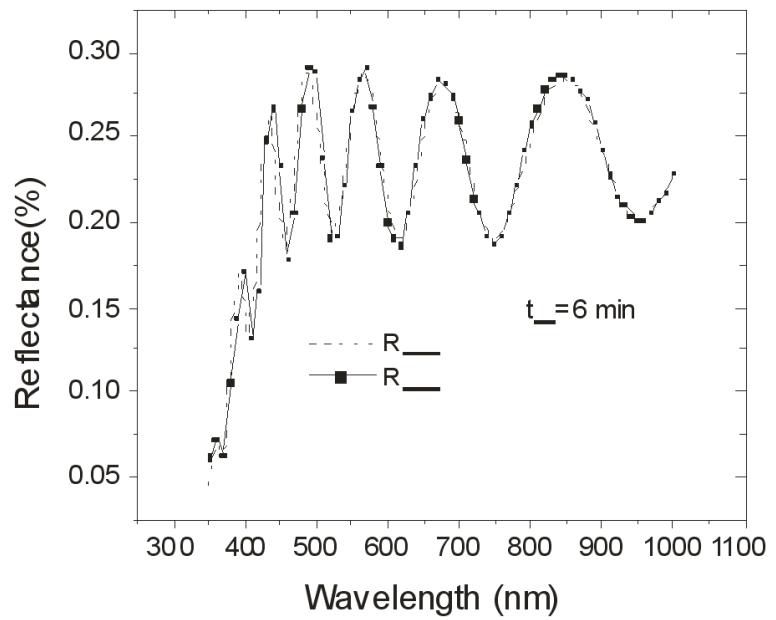

FIG. 5: Reflectance spectrum of a sample prepared on a $1 \Omega$-cm resistivity substrate and at $11.5 \mathrm{~mA} / \mathrm{cm}^{2}$.

a mix of the two, i. e. an effective medium [5]. The porous silicon is considered in the calculation as a homogeneous thin film, deposited on a semi-infinite silicon substrate. The system's reflectance is calculated taking into account the specular reflectance of a homogeneous thin film [7], including a term associated to the sample surface roughness.

The reflectance of the system can then be calculated with the following equation:

$$
R=R_{1} e^{-\left(\frac{4 \pi \sigma}{\lambda}\right)^{2}}
$$

Where $\mathrm{R}$ is the total reflectance of the system, $\mathrm{R}_{1}$ the specular reflectance of a thin film deposited on an absorbent substrate [7], the exponential is the diffused reflectance with normal incident light on the sample; this relation is known as the Davis Relation [8]; $\sigma$ is the rms value of the sample roughness and $\lambda$ is the wavelength of the incident radiation. $R_{1}$ is given by:

$$
R_{1}=\frac{\left(g_{1}^{2}+h_{1}^{2}\right) e^{2 a_{1}}+\left(g_{2}^{2}+h_{2}^{2}\right) e^{-2 \alpha_{1}}+A \cos 2 \gamma_{1}+B \sin 2 \gamma_{1}}{e^{2 \alpha_{2}}+\left(g_{1}^{2}+h_{1}^{2}\right)\left(g_{2}^{2}+h_{2}^{2}\right) e^{-2 \alpha_{1}}+C \cos 2 \gamma_{1}+D \sin 2 \gamma_{1}}
$$

Where

$$
\begin{array}{lc}
g_{1}=\frac{n_{0}^{2}-n_{1}^{2}-k_{1}^{2}}{\left(n_{0}+n_{1}\right)^{2}+k_{1}^{2}} & h_{1}=\frac{2 n_{0} k_{1}}{\left(n_{0}+n_{1}\right)^{2}+k_{1}^{2}} \\
g_{2}=\frac{n_{1}^{2}-n_{2}^{2}+k_{1}^{2}-k_{2}^{2}}{\left(n_{1}+n_{2}\right)^{2}+\left(k_{1}+k_{2}\right)^{2}} & h_{2}=\frac{2\left(n_{1} k_{2}-n_{2} k_{1}\right)}{\left(n_{1}+n_{2}\right)^{2}+\left(k_{1}+k_{2}\right)^{2}} \\
p_{2}=e^{\alpha_{1}} \cos \gamma_{1} & q_{2}=e^{\alpha_{1}} \sin \gamma_{1} \\
t_{2}=e^{-\alpha_{1}}\left(g_{2} \cos \gamma_{1}+h_{2} \sin \gamma_{1}\right), u_{2}=e^{-\alpha_{1}}\left(h_{2} \cos \gamma_{1}-g_{2} \sin \gamma_{1}\right) \\
\alpha_{1}=\frac{2 \pi k_{1} d}{\lambda} \quad \text { and } \quad \gamma_{1}=\frac{2 \pi n_{1} d}{\lambda} \quad(\text { radians }) \\
p_{12}=p_{2}+g_{1} t_{2}-h_{1} u_{2}, & q_{12}=q_{2}+h_{1} t_{2}+g_{1} u_{2} \\
t_{12}=t_{2}+g_{1} p_{2}-h_{1} q_{2}, & u_{12}=u_{2}+h_{1} p_{2}+g_{1} q_{2}
\end{array}
$$

The refraction indexes associated with the PS and the silicon (substrate) are, respectively: $\mathrm{n}_{1}-\mathrm{ik}_{1}$ and $\mathrm{n}_{2}-\mathrm{i} \mathrm{k}_{2}$, being $\mathrm{n}_{1}$ and $\mathrm{n}_{2}$ the real parts of the refraction indexes, $\mathrm{k}_{1}$ and $\mathrm{k}_{2}$ the extinction coefficients. In the statement, $\mathrm{n}_{1}, \mathrm{n}_{2}, \mathrm{k}_{1}, \mathrm{k}_{2}$ depend on the incident radiation wavelength; $\mathrm{n}_{1}, \mathrm{k}_{1}$ and the sample's thickness d, are unknowns which are determine through calculation; $\mathrm{n}_{2}$ and $\mathrm{k}_{2}$ are known and are included in the study as fixed parameters [6].

The theoretical reflectance spectrum is calculated following the procedure described in [9].

Figure 5 Shows the comparison of theoretical and experimental spectra for a sample prepared during an anodizing time of 6 minutes. As observed the theoretical model fits quite well. However, there is a differences of about $12 \%$ in the high absorption region where it is very difficult to carry out the calculation because of the meager information supplied by the experimental spectra. Figures 6 and 7 present the spectral curves for the refraction index and absorption coefficients for samples prepared with different current densities, using a $1 \Omega$-cm substrate.

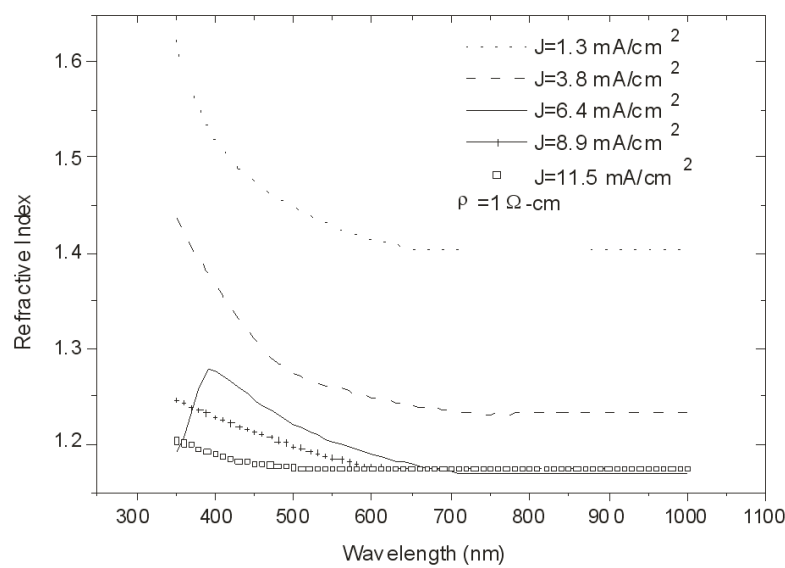

FIG. 6: Refraction index obtained on samples prepared on $1 \Omega$-cm resistivity substrates.

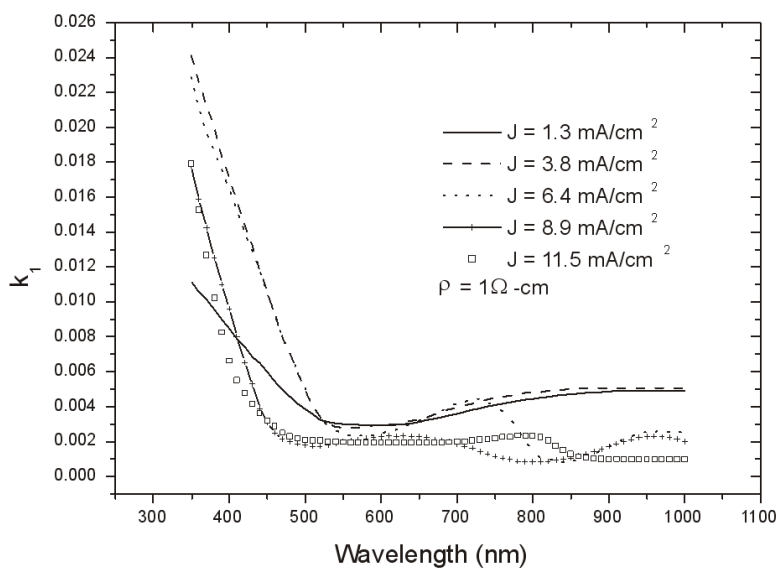

FIG. 7: Extinction coefficients obtained on samples prepared at different anodizing currents.

The index of refraction values are around 1.7 for low current densities and approach 1.1 as the current density in- 
creases. It is well known that the porosity increases with the current density, but those values must be analyzed later on using effective medium theories [5].

Figure 7 presents the calculated absorption coefficients. It is observed that there is absorption in the entire evaluated region, including where the sample was initially supposed to be transparent [9]. This causes the initial index of refraction values to be inaccurate; to amend this, the index of refraction is recalculated after obtaining the absorption coefficient. A shift toward lower wavelengths is also observed for the strong absorption region as the anodizing current is increased. Absorption bands appear in the low absorption region which may be responsible for the PL emission.

Figure 1 shows the thickness obtained trough calculation. Thickness is increased with substrate resistivity and current density. Thickness values were compared with those obtained trough weight measurement and presented a difference of $18 \%$. Roughness values are between 10 and $35 \mathrm{~nm}$.

\section{CONCLUSIONS}

The photoluminescence maxima of samples prepared on $0.01 \Omega$-cm resistivity substrates, at different anodizing cur- rents, are shifted toward shorter wavelengths as the anodizing current increases; The opposite happened for samples prepared on 0.1 and $1 \Omega$-cm substrates. The dependence of the photoluminescence signal on the quantum dimension of the porous silicon samples' skeleton is not discarded, but it is also observed that the signal depends on the fabrication parameters and the substrate resistivity.

A theoretical model is proposed to calculate in a simple way, the optical constants of porous silicon samples deposited on a thick substrate; the model simulates reflectance spectra and allows determining the spectral variation of the refraction index and the absorption coefficient, also it is found the thickness and roughness of the samples.

\section{Acknowledgement}

Authors wish to thank COLCIENCIAS and the International Programme in The Physical Sciences (IPPS), Uppsala University for their economic support to this work.
[1] V. Lehmann and U. Gosele, Apply Phys. Lett. 58, 856 (1991).

[2] V. Grivickas and P. Basmaji, Thin Solid Films 275, 234 (1993).

[3] N. Ookubo, J. Apply Phys. 74, 6375 (1993).

[4] B. Bessais, O. Ben Younes, Ezzaouia, N. Mliki, M. F. Boujmil, M. Oueslati, and R. Bennaceur. J. Lumin. 90, 101 (2000).

[5] J. C Maxwell Garnett, Philos. Trans. R. Soc. London. 203, 385 (1904).

[6] Handbook of Optical Constants of Solids, Edward D. Palik, Aca- demic Press, INC, 1985 p. 563.

[7] Optical properties of Thin Solid Films, O. S. Heavens. Dover Publications, (1991), p. 76.

[8] H. Davies, Proc. IEEE, 101, 209 (1954).

[9] R. Cardenas, J. Torres and J. E. Alfonso, Thin Solid Films, 478, Vol. 1-2 (2005), p. 146-151. 